\title{
Diffuse radio emission in a REFLEX cluster
}

\author{
L. Feretti ${ }^{1}$, P. Schuecker ${ }^{2}$, H. Böhringer ${ }^{2}$, F. Govoni ${ }^{1,3}$, and G. Giovannini ${ }^{1,3}$ \\ 1 Istituto di Radioastronomia - INAF, via Gobetti 101, 40129 Bologna, Italy \\ e-mail: Iferetti@ira.inaf.it \\ 2 Max Planck Institut für Extraterrestrische Physik, PO Box 1312, 85741 Garching, FRG \\ 3 Dipartimento di Astronomia, Univ. Bologna, Via Ranzani 1, 40127 Bologna, Italy
}

Received 3 February 2005 / Accepted 16 July 2005

\section{ABSTRACT}

Deep Very Large Array radio observations are presented for the REFLEX clusters $R X C J 0437.1+0043$ and $R X C J 1314.4-2515$. They are at similar distances and show similar X-ray luminosity, but they are quite different in X-ray structure. Indeed $R X C J 0437.1+0043$ is regular and relaxed, whereas $R X C J 1314.4-2515$ is characterized by substructure and possible merging processes. The radio images reveal the lack of diffuse emission in $R X C J 0437.1+0043$ and the presence of a complex diffuse structure in $R X C J 1314.4-2515$. The diffuse source in the latter cluster consists of a central radio halo which extends to the West toward the cluster periphery and bends to the North to form a possible relic. Another extended source is detected in the eastern cluster peripheral region. Although there could be plausible optical identifications for this source, it might also be a relic candidate owing to its very steep spectrum. The present results confirm the tight link between diffuse cluster radio sources and cluster merger processes.

Key words. galaxies: clusters: general - galaxies: intergalactic medium - radio continuum: general - X-rays: general

\section{Introduction}

Some clusters of galaxies contain extended diffuse radio emission with no optical counterpart, a typical size of $\sim 1 \mathrm{Mpc}$, low surface brightness, and steep radio spectrum. These radio sources are classified as radio halos or relics depending on their central or peripheral position in the cluster, respectively. Radio halos and relics are associated with the existence of cluster merger processes (e.g., Buote 2001; Schuecker et al. 2001; Feretti 2003; Govoni et al. 2004), which provide the energy to reaccelerate the radiating particles. In particular, radio halos originate from particles reaccelerated in the cluster central region, possibly by merger turbulence (Fujita et al. 2003; Brunetti et al. 2004), whereas radio relics are tracers of shock waves produced in the ICM by the flows of cosmological large-scale structure formation (Enßlin et al. 1998; Enßlin \& Gopal-Krishna 2001).

The structure of a radio halo shows close similarity to the X-ray cluster structure (e.g. Deiss et al. 1997; Liang et al. 2000). Moreover in a number of well-resolved clusters, spatial correlation on the large scale between the radio halo brightness and the X-ray brightness is observed (Govoni et al. 2001a), indicating a connection between the non-thermal electrons and thermal intracluster gas. This correlation is also visible in high resolution Chandra data, e.g. for A2744 (Kempner \& David 2004).

The percentage of clusters showing radio halos and relics in the complete X-ray flux limited sample extracted from
Ebeling et al. (1996) is $\simeq 11 \%$. The detection rate increases with the X-ray luminosity up to $\simeq 30 \%$ for the most X-ray luminous clusters (Giovannini \& Feretti 2002). The most powerful radio halos and relics are detected in the clusters with the highest X-ray luminosity and the largest total mass, as derived from the radio power-X-ray luminosity and radio power-cluster mass correlations (Liang et al. 2000; Bacchi et al. 2003; Govoni et al. 2001b; Giovannini \& Feretti 2004). The connection between radio halos and cluster mergers is confirmed by the radio spectra in A665 and A2163, which are flatter in the regions influenced by merger processes (Feretti et al. 2004).

According to the previous findings, we expect radio halos and relics to be common in X-ray luminous clusters that have irregular X-ray morphology. Schuecker \& Böhringer (1999) show that the ROSAT All Sky Survey (RASS) might be helpful for finding good cluster candidates housing radio halos. The list of clusters from the RASS with X-ray luminosity $L_{\mathrm{X}}>10^{45} \mathrm{erg} \mathrm{s}^{-1}$, computed assuming $H_{0}=50 \mathrm{~km} \mathrm{~s}^{-1} \mathrm{Mpc}^{-1}$ and $q_{0}=0.5$, therefore provides a list of promising clusters that host a radio halo, detectable if observed with enough sensitivity.

A well-selected sample of X-ray cluster sources found in the southern ROSAT All-Sky Survey has been obtained as a result of the REFLEX cluster survey (Böhringer et al. 2001, 2004). We inspected the images of the NRAO VLA Sky Survey (NVSS; Condon et al. 1998) for the 20 most X-ray luminous clusters in the overall REFLEX catalogue of 447 clusters and 

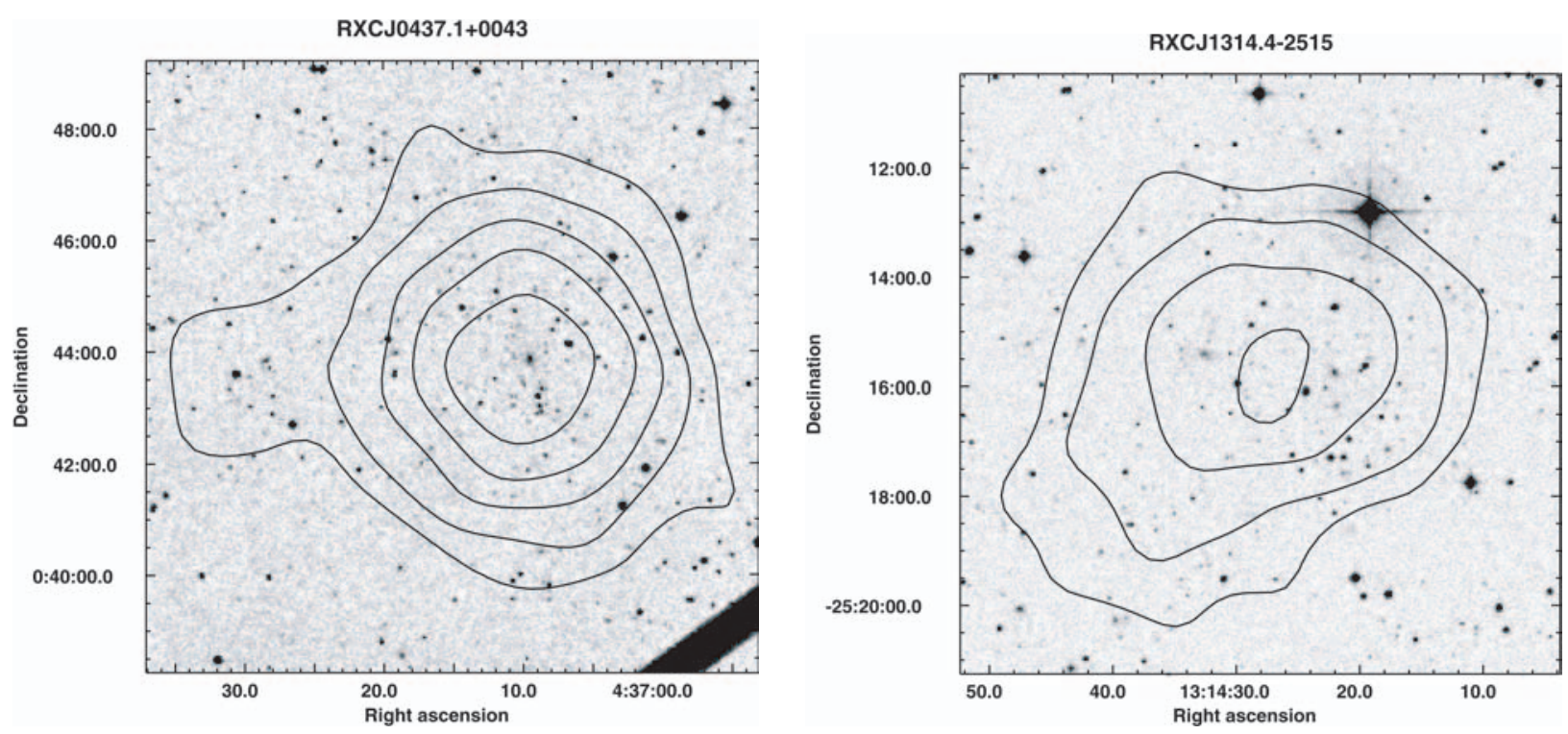

Fig. 1. Contour X-ray images from ROSAT All Sky Survey in the $0.5-2 \mathrm{keV}$ energy range of the two clusters $R X C$ J0437.1+0043 (left panel) and $R X C J 1314.4-2515$ (right panel), overlapped onto the grey scale optical images from the Digital Sky Survey.

found hints of diffuse radio emission for $R X C J 0437.1+0043$ and $R X C J 1314.4-2515$, in addition to some well known clusters also belonging to the Abell catalogue.

The cluster $R X C J 0437.1+0043$ is at redshift 0.2842 and has an X-ray luminosity of $8.99 \times 10^{44} \mathrm{erg} \mathrm{s}^{-1}$ in the $0.1-2.4 \mathrm{keV}$ band $^{1}$. The cluster $R X C J 1314.4-2515$ is at redshift 0.2439 and has an X-ray luminosity of $1.09 \times 10^{45} \mathrm{erg} \mathrm{s}^{-1}$ in the $0.1-2.4 \mathrm{keV}$ band. These two clusters are presented in Fig. 1, where the low resolution X-ray images from the ROSAT All Sky Survey are overlayed on the grey scale optical images taken from the Digital Sky Survey.

We obtained sensitive radio observations at $1.4 \mathrm{GHz}$ with the Very Large Array (VLA), with the aim of searching for diffuse radio sources in these clusters. To properly map these diffuse sources, high sensitivity for extended low brightness feature is needed, but a good resolution is also necessary to distinguish a real diffuse source from the blend of unrelated sources.

The similarity between the radio and the X-ray morphology, the high cluster X-ray temperature and luminosity, and the evidence of substructure in clusters with radio halos all indicate the importance of also considering the cluster X-ray properties. Here we present new radio images of these clusters and compare them with the X-ray emission.

In Sect. 2 we present the radio data reduction. In Sects. 3 and 4 we give the results for the two clusters $R X C J 0437.1+0043$ and $R X C J 1314.4-2515$, respectively. The discussion and conclusions are reported in Sect. 5.

With the adopted cosmology, the angular size of 1 arcsec corresponds to $4.29 \mathrm{kpc}$ at the distance of $R X C J 0437.1+0043$ and to $3.84 \mathrm{kpc}$ at the distance of $R X C J 1314.4-2515$.

\footnotetext{
1 Throughout the paper we use the $\Lambda$ CDM cosmology with $H_{0}=$ $70 \mathrm{~km} \mathrm{~s}^{-1} \mathrm{Mpc}^{-1}, \Omega_{\mathrm{m}}=0.3$, and $\Omega_{\Lambda}=0.7$.
}

\section{Radio data}

Deep radio observations were obtained for the two clusters with more compact configurations of the VLA as presented in Table 2. In order to detect low brightness emission, a good sampling of short spacings is necessary. The shortest baseline in the observations is of $35 \mathrm{~m}$, corresponding to $200 \lambda$. This ensures that a large scale structure of size up to $\sim 7.5^{\prime}$ is properly imaged. On the other hand, the relatively good angular resolution supplied by the longest baselines is important to separate discrete radio sources. For the observations of the southern cluster $R X C J 1314.4-2515$, the VLA was used in hybrid configurations $(\mathrm{CnB}$ and $\mathrm{DnC})$, in order to obtain a roughly circular restoring beam.

For the cluster $R X C J 0437.1+0043$, the sources $3 C 48$ and J0423-013 were observed as calibrators of the flux density scale, and of the antenna gains and phases, respectively. For $R X C J 1314.4-2515$, the source 3C 286 was observed for the flux density scale calibration, and the source J1284-199 was observed as gain and phase calibrator.

The data were calibrated and reduced with the Astronomical Image Processing System (AIPS), following the standard procedure. Significant editing of the uv data was needed to identify and remove bad data. Images were produced by Fourier-Transform, Clean and Restore, using the task IMAGR with uniform uv weight, no Gaussian taper, and ROBUST $=0$. Several cycles of imaging and self-calibration were performed, to minimize the effects of amplitude and phase variations. At each step, clean components were carefully selected as a model for the self-calibration. Gain calibration solutions were obtained with a long integration time only after a few cycles of phase calibration. The process was stopped when no further significant improvement was obtained. Then, a deep cleaning was applied to the data, until the total cleaned flux reached a stable value. 
Table 1. Details of the VLA observations.

\begin{tabular}{cccccc}
\hline \hline $\begin{array}{c}\text { Name } \\
\text { RXC J }\end{array}$ & $\begin{array}{c}\text { Frequency } \\
\text { MHz }\end{array}$ & $\begin{array}{c}\text { Bandw. } \\
\text { MHz }\end{array}$ & Config. & $\begin{array}{c}\text { Obs. time } \\
\text { hours }\end{array}$ & Date \\
\hline $0437.1+0043$ & $1365 / 1465$ & 50 & $\mathrm{C}$ & 2.4 & Jun. 2000 \\
& $1365 / 1465$ & 50 & $\mathrm{D}$ & 2.4 & Aug. 2000 \\
\multirow{2}{*}{$1314.4-2515$} & $1365 / 1465$ & 50 & $\mathrm{CnB}$ & 2.4 & Mar. 2000 \\
& $1365 / 1465$ & 50 & DnC & 2.4 & Jul. 2000 \\
\hline
\end{tabular}

Col. 1: cluster name; Col. 2: observing frequency; Col. 3: bandwidth; Col. 4: VLA configuration;

Col. 5: observing time; Col. 6: observing date.

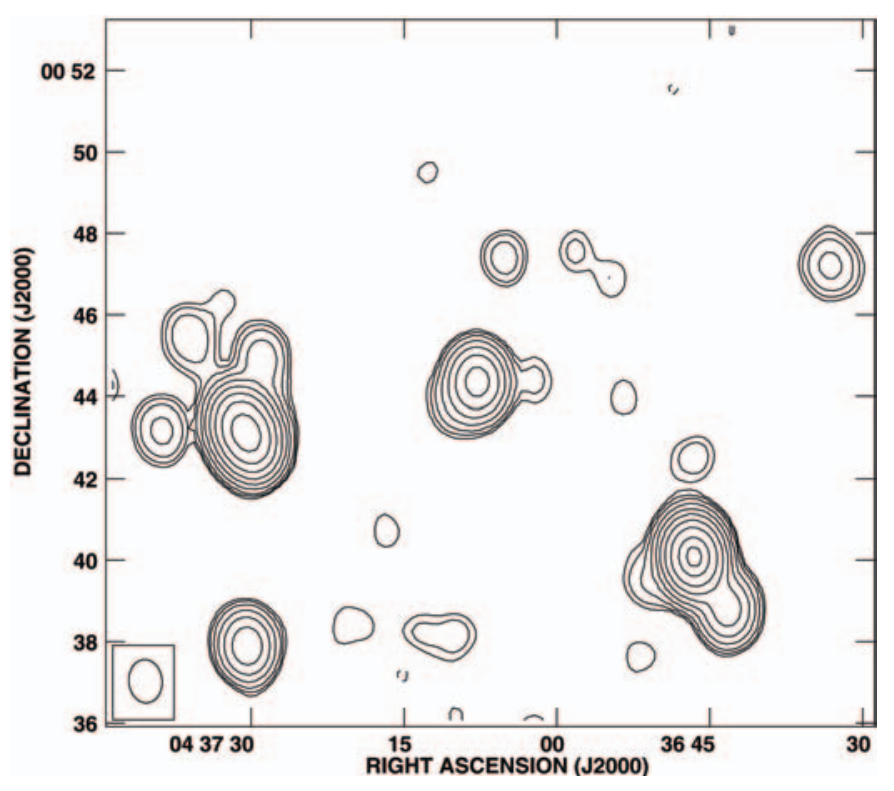

Fig. 2. Contour radio image of the cluster $R X C J 0437.1+0043$ obtained with the D array at $1.4 \mathrm{GHz}$, with resolution of $63.9^{\prime \prime} \times 48.9^{\prime \prime}$ $\left(F W H M\right.$ at $\left.\mathrm{PA}=5^{\circ}\right)$. The rms noise level is $0.06 \mathrm{mJy} /$ beam. Contour levels are $-0.2,0.2,0.3,0.5,1,2,4,8,16,32,48 \mathrm{mJy} / \mathrm{beam}$. In this and the following images, the observing beam is indicated at the bottom left corner.

Data from the two different configurations were reduced separately, in order to analyse the possible existence of spurious features. Images at intermediate resolutions were also obtained by adding together the data from the two configurations.

The achieved sensitivities in the low-resolution images are $0.06 \mathrm{mJy} /$ beam $R X C J 0437.1+0043$ and $0.068 \mathrm{mJy} /$ beam in $R X C J 1314.4-2515$. The confusion level expected in the VLA images in the D configuration is $\sim 70 \mu \mathrm{Jy} / \mathrm{beam}$ (J. Condon and R. Perley, private communication); thus, in our images we reach the confusion limit. Calibration of the polarized emission was not possible because of technical problems. Therefore, polarization information cannot be derived from the present radio data.

\section{Results on RXC J0437.1+0043}

This cluster belongs to the REFLEX-DXL sample defined by Zhang et al. (2004). In the X-ray image, obtained with $\mathrm{XMM}$ in the $0.5-2 \mathrm{keV}$ energy range (Böhringer et al. 2005,

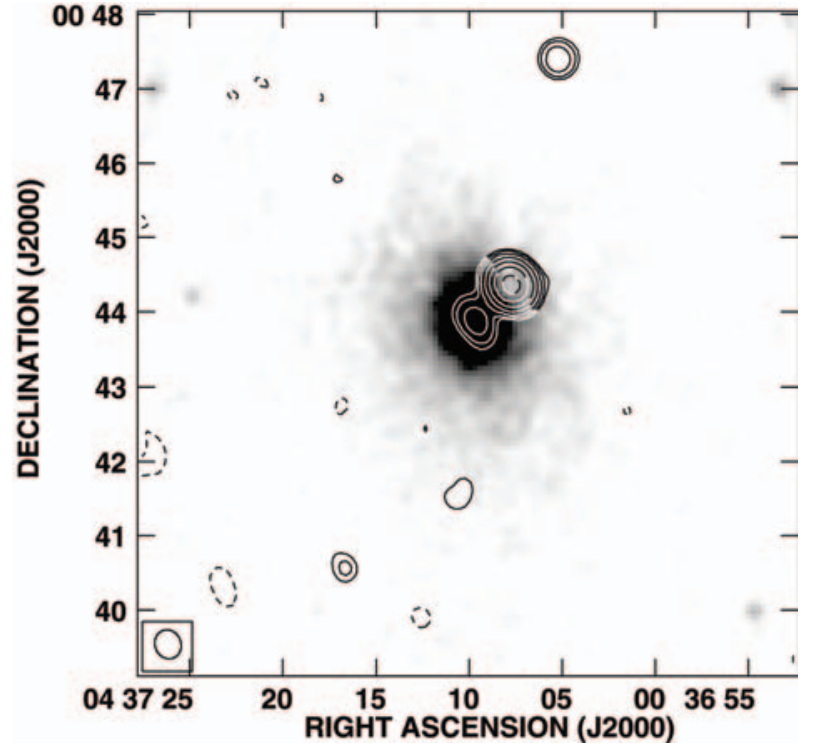

Fig. 3. Contour radio image of the cluster RXC J0437.1+0043 obtained at $1.4 \mathrm{GHz}$ with the $\mathrm{C}$ array, overlayed on the grey scale X-ray emission detected by XMM. The FWHM is $23.7^{\prime \prime} \times 20.9^{\prime \prime}$ (at $\mathrm{PA}=23^{\circ}$ ). The rms noise level is $0.06 \mathrm{mJy} /$ beam. Contour levels are $-0.2,0.2,0.3,0.5,1,2,4,8 \mathrm{mJy} /$ beam.

in preparation), the cluster looks relatively symmetric with some elongation. It has a very bright center, with the temperature falling from about $7-8 \mathrm{keV}$ in the outer region to about $5 \mathrm{keV}$ in the center. Thus it is very likely a relaxed cluster that shows a cooling core.

The radio emission of the cluster, as detected at low resolution, is presented in Fig. 2. The field shown in the figure covers a much larger cluster area than shown in the left panel of Fig. 1. There are several discrete radio sources, and no evidence of any large scale diffuse emission. The higher resolution image of the central cluster region, obtained with the $\mathrm{C}$ array, is shown in Fig. 3, overlayed on the grey scale X-ray emission. The only radio emission at the center of this cluster is represented by two close unresolved radio sources of 1.1 and $11.6 \mathrm{mJy}$, respectively, one coincident with the X-ray peak, the other slightly displaced from it (see Table 2). Both sources are likely to be identified with galaxies (see Fig. 4 and Table 2).

No diffuse radio emission is detected in this cluster at the brightness level of $0.18 \mathrm{mJy} /$ beam $(3 \sigma)$, corresponding to $\sim 0.06 \mu \mathrm{Jy} \operatorname{arcsec}^{-2}$. This value is lower than the average 
Table 2. Radio sources at the center of RXC J0437.1+0043.

\begin{tabular}{|c|c|c|c|}
\hline Radio & \multicolumn{3}{|c|}{ Optical } \\
\hline RA (2000) Dec & $S_{1.4 \mathrm{GHz}}$ & RA (2000) Dec & $m_{B}$ \\
\hline $\mathrm{h} m \mathrm{~s} \circ, " \prime$ & mJy & $\mathrm{h} m \mathrm{~s} \circ, \prime \prime$ & \\
\hline $043707.8+004423$ & $11.6 \pm 0.2$ & $043707.88+004422.8$ & 21.0 \\
\hline $043709.6+004355$ & $1.1+0.1$ & $043709.49+004351.1$ & 18.9 \\
\hline
\end{tabular}

Col. 1: radio source position; Col. 2: flux density at $1.4 \mathrm{GHz}$;

Col. 3: optical position from superCOSMOS; Col. 4: blue magnitude.

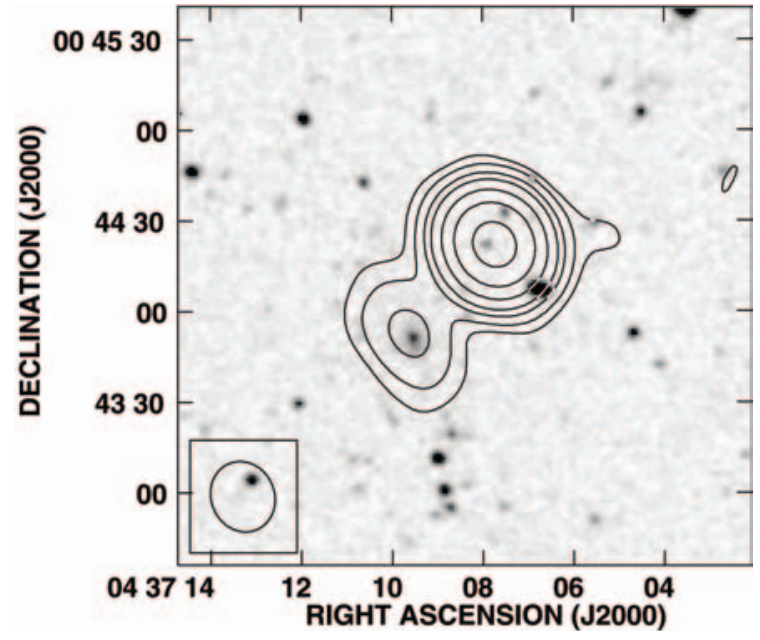

Fig. 4. Contour radio image of the cluster $R X C J 0437.1+0043$ from Fig. 3, superposed on the grey-scale optical image from the DSS2 red filter. Contour levels are 0.15, 0.3, 0.6, 1, 2, 4, 8 mJy/beam.

brightness of the radio halo Coma $\mathrm{C}$ in the Coma cluster. The maximum size of an extended feature detectable with the present observations is $\sim 7.5^{\prime}$, which corresponds to a linear size of $\sim 1.9 \mathrm{Mpc}$. Thus a radio halo with a regular structure, similar in brightness to Coma $\mathrm{C}$, and extended $<1.9 \mathrm{Mpc}$ would have been easily detected by our observations.

\section{Results on RXC J1314.4-2515}

\subsection{Large-scale cluster radio emission}

The radio image presented in Fig. 5 was obtained with the data of the DnC array with a deep cleaning down to $0.07 \mathrm{mJy} /$ beam, which corresponds approximately to the image noise level. The figure shows a large field down to about $50 \%$ of the antenna primary beam, with the lowest positive contour corresponding to 3 times the noise level. Diffuse emission is easily visible at the cluster center, with a brightness of about 10 times the noise level. No other features, either negative or positive, are present in the image at the same level, ensuring that the diffuse emission cannot be an artifact of the data reduction process.

Figure 6 shows enlargement of the central cluster region, with the radio emission overlayed on the optical image.

In Fig. 7 we present an image at intermediate resolution obtained from the combination of the data from the DnC and $\mathrm{CnB}$ arrays, using UVTAPER $=10$ and $\mathrm{ROBUST}=0$ with the AIPS task IMAGR. A deep cleaning down to about $1 \sigma$ level

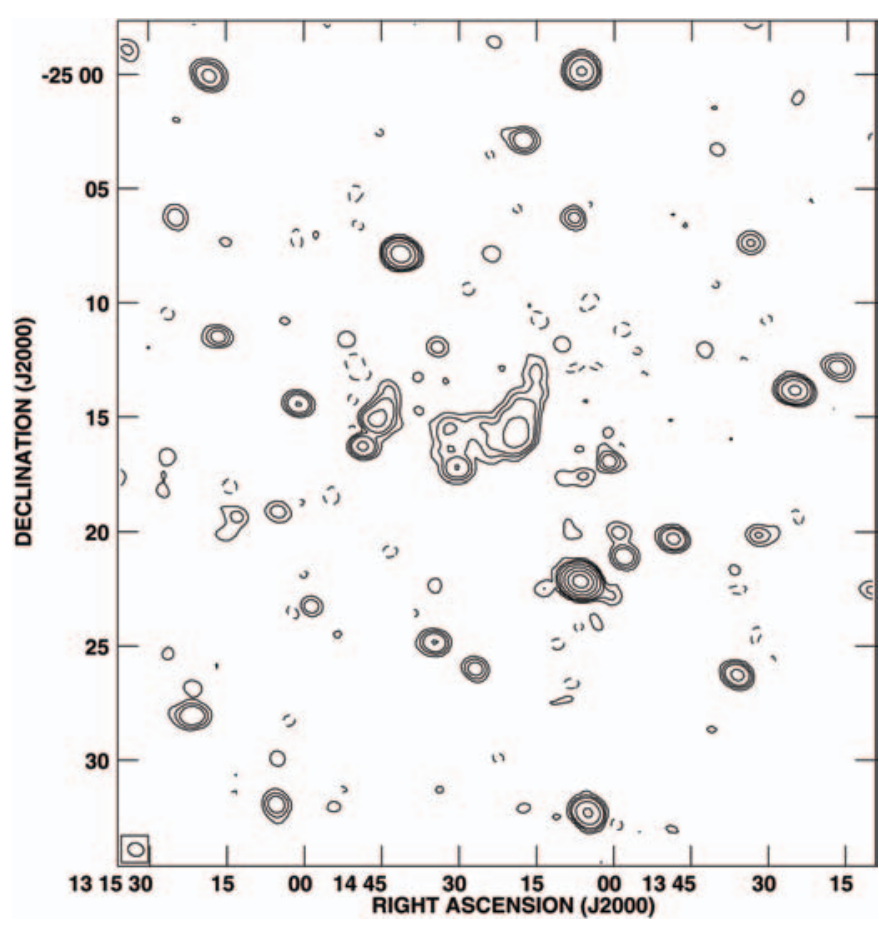

Fig. 5. Radio image of the cluster RXCJ1314.4-2515 obtained at $1.4 \mathrm{GHz}$ with the DnC array at the resolution of $41.8^{\prime \prime} \times 34.0^{\prime \prime}$ (at $\mathrm{PA}=75^{\circ}$ ). The rms noise level is $0.068 \mathrm{mJy} / \mathrm{beam}$. Contour levels are $-0.5,-0.2,0.2,0.5,1.0,3.0,10.0,30.0,80.0 \mathrm{mJy} / \mathrm{beam}$.

was applied. The two images agree very closely, confirming the diffuse nature of the extended emission detected at lower resolution. The slightly higher noise level in the intermediate resolution image with respect to the low resolution image is probably due to the lower weight of the shortest baselines, where most of the diffuse flux density is present.

The diffuse radio emission in this cluster is complex. The most relevant feature (labeled $\mathrm{H}$ in Fig. 6) permeates the cluster center, around the brightest cluster galaxy, and is elongated approximately in E-W direction. Its brightest region is on the western side (R1) and coincides with a prominent bend to the North. The total flux density of this emission is given in Table 3 . Its total extent is at least $8^{\prime}$, corresponding to $\sim 1.8 \mathrm{Mpc}$. The point source at the southern boundary of the diffuse feature $(\mathrm{S})$ is an unrelated discrete source (see the higher resolution image of Fig. 8). Another extended source showing an elongated structure (R2 in Fig. 6) is detected in the eastern cluster region. 
Table 3. Extended radio sources in RXC J1314.4-2515.

\begin{tabular}{|c|c|c|c|c|}
\hline \multirow{3}{*}{$\begin{array}{l}\text { Radio } \\
\text { Source }\end{array}$} & \multirow{3}{*}{$\begin{array}{c}S_{1.4 \mathrm{GHz}} \\
\text { DnC arr } \\
\text { mJy }\end{array}$} & \multirow{3}{*}{$\begin{array}{c}S_{1.4 \mathrm{GHz}} \\
\mathrm{CnB} \text { arr } \\
\mathrm{mJy}\end{array}$} & Optical & \multirow{3}{*}{$m_{B}$} \\
\hline & & & RA (2000) Dec & \\
\hline & & & $\mathrm{h} \mathrm{m} \mathrm{s}$ & \\
\hline $\mathrm{H}+\mathrm{R} 1$ & $40.5 \pm 0.6$ & & & \\
\hline \multirow[t]{3}{*}{$\mathrm{R} 1$} & & $20.2 \pm 0.5$ & $131419.60-251538.0$ & 16.1 \\
\hline & & & $131419.30-251530.0$ & 20.1 \\
\hline & & & $131416.86-251558.9$ & 18.1 \\
\hline \multirow[t]{2}{*}{$\mathrm{R} 2$} & $11.1 \pm 0.3$ & $10.1 \pm 0.3$ & $131444.54-251436.7$ & 18.2 \\
\hline & & & $131446.19-251509.0$ & 21.4 \\
\hline
\end{tabular}

Col. 1: source name; Col. 2: $1.4 \mathrm{GHz}$ flux density at lower resolution;

Col. 3: $1.4 \mathrm{GHz}$ flux density at higher resolution;

Col. 4: position of optical objects from superCOSMOS; Col. 5: blue magnitude.

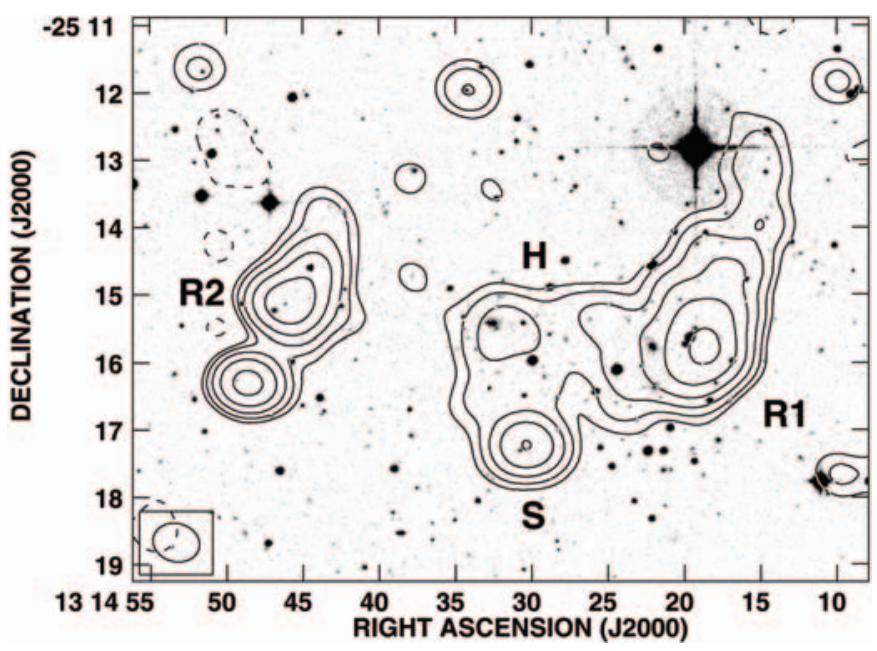

Fig. 6. Contour radio emission of the central region of RXC J1314.4-2515 overlayed on the grey-scale red image from the DSS2. The radio image, obtained from the DnC array data, has a resolution of $41.8^{\prime \prime} \times 34.0^{\prime \prime}\left(F W H M\right.$ at $\left.\mathrm{PA}=75^{\circ}\right)$ and a noise level of $0.068 \mathrm{mJy} / \mathrm{beam}$. Contour levels are $-0.2,0.2,0.4,0.8,1.6,3.2$, $6.4 \mathrm{mJy} /$ beam. Relevant regions are marked by labels (see text).

\subsection{Sources $R 1$ and $R 2$}

The high-resolution image of the cluster central region, obtained with the $\mathrm{CnB}$ array data, is shown in Fig. 8. Most of the extended structure was resolved out, confirming that it is really diffuse and cannot be due to discrete sources. The only discrete source detected within the central region of the diffuse emission is very faint, with a total flux of $\sim 0.5 \mathrm{mJy}$, and is identified with the brightest cluster galaxy (BCG in Fig. 8), in agreement with Valtchanov et al. (2002). No jet-like structure is visible.

The region of highest brightness $\mathrm{R} 1$ is detected as a feature with amorphous morphology and a steep brightness gradient on the S-W edge. This source accounts for about $50 \%$ of the flux density of the whole diffuse emission detected at low resolution (Table 3). The diffuse source R2 is resolved in at least 3 components, with an overall structure reminiscent of

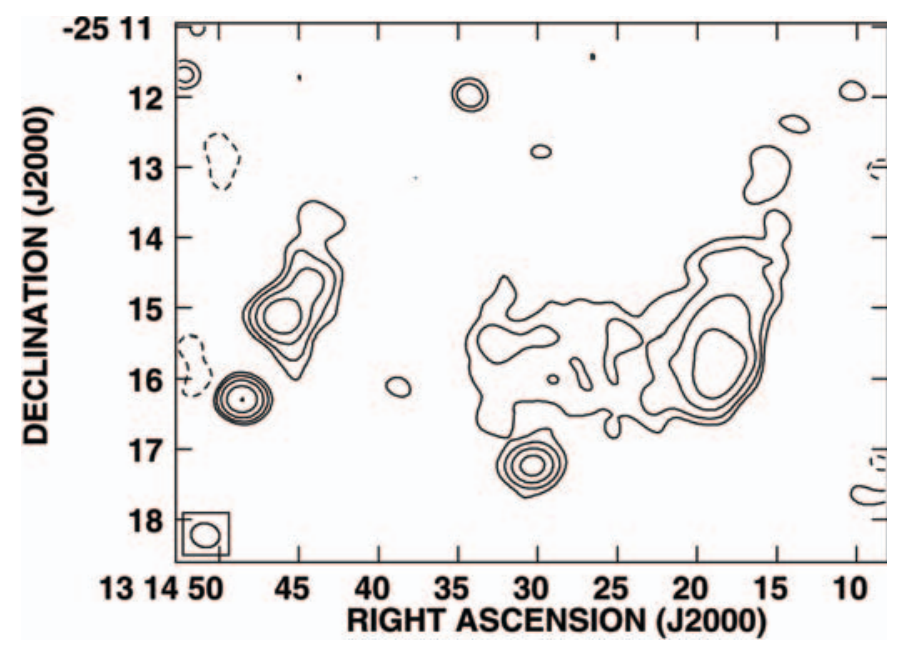

Fig. 7. Radio image of the cluster $R X C J 1314.4-2515$ at $1.4 \mathrm{GHz}$ obtained with the combined $\mathrm{CnB}$ and $\mathrm{DnC}$ array, with resolution of $24.4^{\prime \prime} \times 20.4^{\prime \prime}$ (at $\mathrm{PA}=77.9^{\circ}$ ). The rms noise level is $0.09 \mathrm{mJy} / \mathrm{beam}$. Contour levels are $-0.27,0.27,0.5,1,2,4,8$ mJy/beam.

that of a wide-angle tailed (WAT) radio source; however, its central component is likely extended.

A radio image of this cluster obtained at $1.4 \mathrm{GHz}$ with the Australia Telescope Compact Array (ATCA) in the 6C configuration with a resolution of $23.8^{\prime \prime} \times 9.4^{\prime \prime}$ is presented by Valtchanov et al. (2002). This image is very similar to our high resolution image of Fig. 8. Also in the ATCA image, as in Fig. 8, the lower brightness emission is missing. This is consistent with the fact that this emission is diffuse, so that a more compact ATCA configuration than the $6 \mathrm{C}$ configuration would be needed to detect it.

Valtchanov et al. (2002) identify the faint radio source at the center with the brightest cluster galaxy (BCG) and discuss the possibility that R1 and R2 are the symmetric lobes of an FRII radio galaxy with the core coincident with the BCG. However, they consider this interpretation quite questionable, as the radio galaxy is not located in an underdense environment as expected for such a giant radio galaxy. 


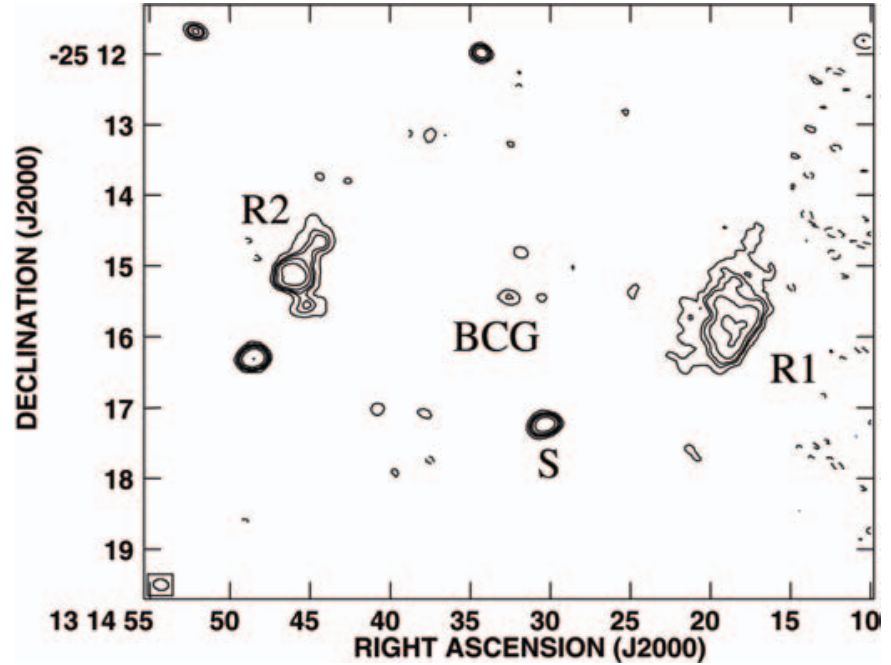

Fig. 8. Radio image of the cluster $R X C J 1314.4-2515$ at $1.4 \mathrm{GHz}$ obtained with the $\mathrm{CnB}$ array with resolution of $12.8^{\prime \prime} \times 9.3^{\prime \prime}$ (at $\mathrm{PA}=88.5^{\circ}$ ). The rms noise level is $0.045 \mathrm{mJy} /$ beam. Contour levels are $-0.15,0.15,0.3,0.4,0.6,1,3,6,10,20,50,80 \mathrm{mJy} / \mathrm{beam}$.

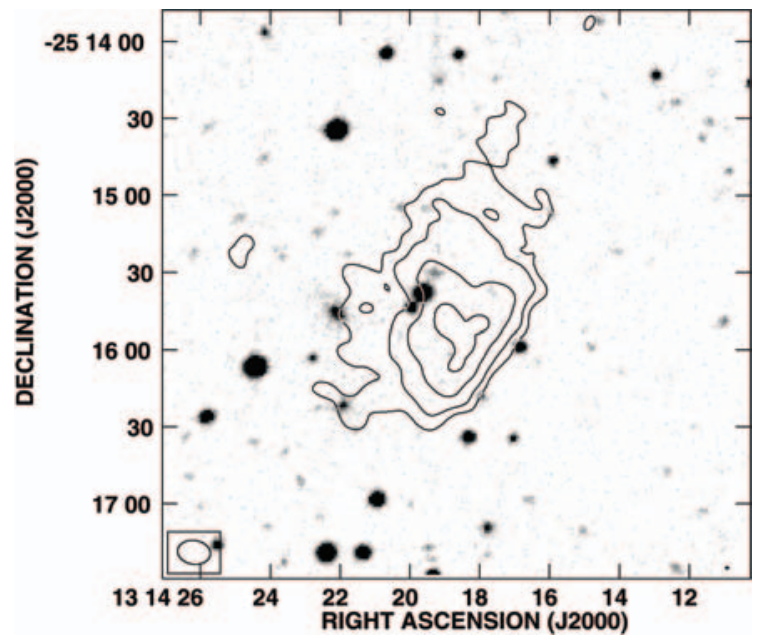

Fig. 9. Enlargement of the region R1 of Fig. 8, overlayed on the greyscale optical image taken from the DSS2 red filter. Contour levels are $0.15,0.3,0.6,1 \mathrm{mJy} /$ beam.

We have searched if there are plausible identifications for sources R1 and R2. The positions and magnitudes of the optical candidates are given in Table 3. From the radio-optical overlay presented in Fig. 9, it turns out that the source R1 is not obviously associated with any optical galaxy. Among the objects listed in Table 3, i.e. those well within the radio contours, the brightest object is a star (Valtchanov et al. 2002).

The radio-optical overlay of the source R2 (Fig. 10) shows that two optical objects could be responsible for the radio emission (see Table 3): a faint galaxy coincident with the northern component and a fainter galaxy located approximately at the peak of the middle component. In the first case, the source would be a tailed radio galaxy, but one showing an unusual structure with a very bright region at the center of the tail. In the second case, the source would be a WAT radio galaxy, but this seems unlikely, since WAT sources are usually located at

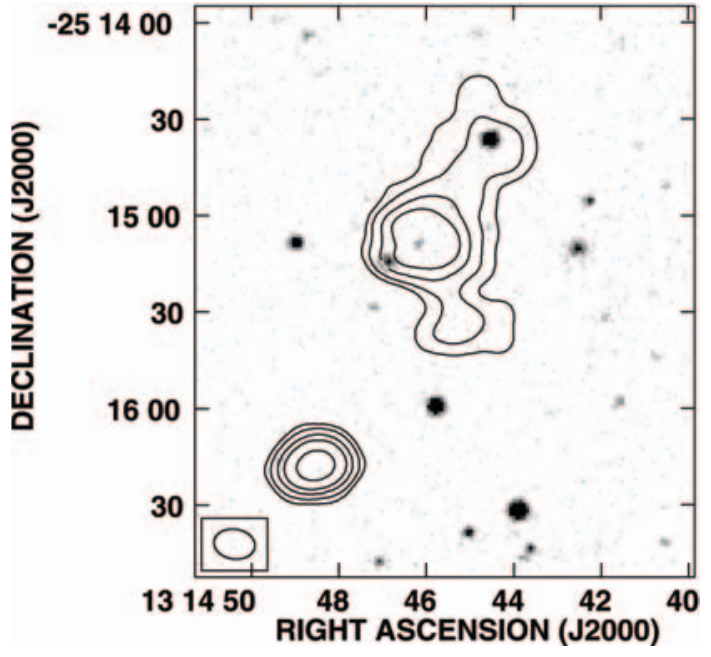

Fig. 10. Enlargement of the region R2 of Fig. 8, overlayed on the greyscale optical image taken from the DSS2 red filter. Contour levels are $0.15,0.3,0.6,1,2 \mathrm{mJy} /$ beam.

cluster centers. Alternatively, if the radio emission were due to both optical objects, the northern radio galaxy would be slightly extended, and the other radio galaxy, consisting of the central and southern components of R2, would show a tailed structure. The spectral information, available from Valtchanov et al. (2002), does not seem to support the identification of the source R2 with radio galaxies. Indeed, those authors derive a very steep spectrum with $\alpha_{1.4}^{2.5} \sim 2.5\left(S_{v} \propto v^{-\alpha}\right)$, and suggest that this source is an excellent candidate for a relic source.

\subsection{Radio-X-ray comparison}

The large-scale X-ray emission of this cluster (Fig. 1, right panel) shows an asymmetric brightness distribution with a substructure to the S-E, which is an indication of a recent cluster merger. An ongoing merger at the cluster center is also derived by the ROSAT HRI image (Valtchanov et al. 2002, and Fig. 11), which shows emission clearly elongated approximately in the E-W direction. From the overlay between the radio and X-ray HRI emission (Fig. 11), it is evident that the diffuse radio emission permeates the cluster center and extends toward the West to the cluster periphery, then bends to the North.

On the basis of the typical classification of radio halos and relics in clusters, the diffuse radio emission at the cluster center, coincident with the region of highest X-ray brightness, can be classified as a radio halo with irregular and elongated morphology. The diffuse structure, including source R1 and extending toward the North, could be classified as a relic. This structure has a total size of $\sim 4^{\prime}$, i.e. about $900 \mathrm{kpc}$. It is located at $\sim 3.5^{\prime}$ from the cluster center, i.e. at a projected distance of about $800 \mathrm{kpc}$. We note that this peripheral diffuse emission is significantly more extended toward the North than region R1 imaged at higher resolution and classified as a relic by Valtchanov et al. (2002).

On the other hand, since the diffuse emission looks like a single radio feature, we could consider it as a very unusual and peculiar cluster radio halo. From the total flux density 


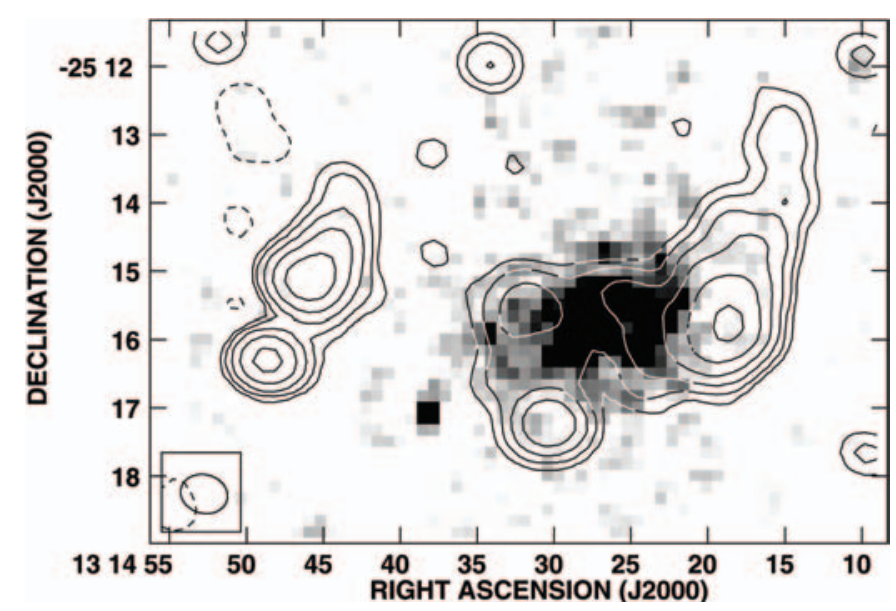

Fig. 11. Contour radio image of the cluster $R X C J 1314.4-2515$ at $1.4 \mathrm{GHz}$, superposed on the grey scale X-ray emission obtained by ROSAT HRI. Contour levels are $-0.2,0.2,0.4,0.8,1.6,3.2$, $6.4 \mathrm{mJy} /$ beam.

at $1.4 \mathrm{GHz}$ of the extended feature $\mathrm{H}$, including the peripheral structure (Table 3 ), a monochromatic radio power of $7.3 \times$ $10^{24} \mathrm{~W} \mathrm{~Hz}^{-1}$ is obtained for this source. This is about 10 times higher than the power of the radio halo in Coma, and is comparable to that of the most powerful halos in hot clusters. Thus, on the basis of the power, we cannot exclude the possibility that the whole feature is a very unusual radio halo. More data are necessay to clarify this point and check if this source is consistent with the radio power- X-ray luminosity correlation (Bacchi et al. 2003; Govoni et al. 2001b).

The western source $\mathrm{R} 2$ is located in a peripheral cluster region at a projected distance of $\sim 800 \mathrm{kpc}$ from the cluster center and has a total extent of about $500 \mathrm{kpc}$ with the major axis roughly perpendicular to the cluster radius. Valtchanov et al. (2002) suggest that it is a candidate for a relic source owing to its extent, location, and very steep spectrum. From our data, it is not possible to firmly establish its nature. We find that it may have plausible optical identifications; however, more detailed information on its structure and spectrum are needed to understand if this emission is due to a relic or to discrete radio galaxies.

\section{Discussion and conclusions}

The two clusters studied here have similar redshift and X-ray luminosity, but show different X-ray structure. The cluster $R X C J 0437.1+0043$ is rather regular and relaxed and is characterized by a cooling core, whereas the cluster $R X C J 1314.4-2515$ shows substructure to the S-E on the large scale and elongation in the E-W direction in the innermost region, thus showing merging activity.

Diffuse emission on a scale of $\sim 1.8 \mathrm{Mpc}$ is detected in $R X C J 1314.4-2515$. This emission is rather irregular in shape, extending to the peripheral western cluster boundary and bending to the North. We suggest that this morphology is likely to consist of a central radio halo and a peripheral relic. The two diffuse sources are strictly connected to each other, making this diffuse emission quite peculiar. The only cluster showing a similar complex diffuse emission is A754 (Kassim et al. 2001; Bacchi et al. 2003), where there is a central halo and a more peripheral relic, which show atypical structures and may be connected to each other.

The elongation of the radio structure at the cluster center is in the same direction of the X-ray brightness distribution detected at high resolution by the ROSAT HRI. Since the E-W elongation of the X-ray brightness distribution probably indicates a cluster merger in that direction, the radio structure confirms the existence of a tight connection between the radio halo and the central merger. The source $\mathrm{R} 1$ is located along the major axis of the HRI X-ray brightness distribution, i.e. along the merger axis, where merger shocks are expected. Its structure is elongated nearly perpendicular to the merger axis. In the framework of models that suggest relics as tracers of cluster merger shock waves (Enßlin et al. 1998; Enßlin \& Gopal-Krishna 2001), both the location and the structure of R1 seem to be consistent with this source being a cluster relic.

In addition to the diffuse emission discussed above, there is also the extended radio emission $\mathrm{R} 2$ in the eastern cluster region, which may be a candidate for another relic source (see also Valtchanov et al. 2002). We could speculate that the possible existence of a second cluster relic would be consistent with the dynamical activity derived in this cluster. Indeed, the two relics would be located at opposite sides of the cluster, along the merger axis with their structure elongated roughly perpendicularly to the merger axis. In this respect, cluster RXC J1314.4-2515 would be similar to A3667 (Röttgering et al. 1997), although in cluster A3667, the two opposite relics are much more extended and no central halo is detected.

The presence of diffuse radio sources in RXC J1314.4-2515, together with the lack of a radio halo in $R X C J 0437.1+0043$, confirms that the formation of diffuse emission is very likely to be associated with the cluster merger activity. A consistent, although qualitative, picture is that $R X C J 1314.4-2515$ exhibits a central halo linked to the central merger, and relic emission originating from the peripheral shocks. Future sensitive and high resolution data both in radio and $\mathrm{X}$-ray are needed to better investigate the properties of the cluster $R X C J 1314.4-2515$ and to study the details of the connection between merger processes and the formation of halos and relics.

Acknowledgements. We thank the anonymous referee for his/her suggestions, which have significantly improved presentation of the results. NRAO is a facility of the National Science Foundation, operated under cooperative agreement by Associated Universities, Inc. This work was partly supported by the Italian Space Agency (ASI).

\section{References}

Bacchi, M., Feretti, L., Giovannini, G., \& Govoni, F. 2003, A\&A, 400, 465

Böhringer, H., Schuecker, P., Guzzo, L., et al. 2001, A\&A, 369, 826

Böhringer, H., Schuecker, P., Guzzo, L., et al. 2004, A\&A, 425, 367

Brunetti, G., Blasi, P., Cassano, R., \& Gabici, S. 2004, MNRAS, 350, 1174

Buote, D. A. 2001, ApJ, 553, L15

Condon, J. J., Cotton, W. D., Greisen, E. W., et al. 1998, AJ, 115, 1693 
Deiss, B. M., Reich, W., Lesch, H., \& Wielebinski, R. 1997, A\&A, 321,55

Ebeling, H., Voges, W., Böhringer, H., et al. 1996, MNRAS, 281, 799

Enßlin, T. A., \& Gopal-Krishna 2001, A\&A, 366, 26

Enßlin, T. A., Biermann, P. L., Klein, U., \& Kohle, S. 1998, A\&A, 332,395

Feretti, L. 2003, in Texas in Tuscany, XXI Symp. on Relativistic Astrophysics, ed. R. Bandiera, R. Maiolino, \& F. Mannucci (Singapore: World Sci. Publ.), 209

Feretti, L., Orrú, E., Brunetti, G., et al. 2004, A\&A, 423, 111

Fujita, Y., Takizawa, M., \& Sarazin, C. L. 2003, ApJ, 584, 190

Giovannini, G., \& Feretti, L. 2002, in Merging Processes of Galaxy Clusters, ed. L. Feretti, I. M. Gioia, \& G. Giovannini, ASSL (Kluwer Ac. Publish.), 197

Giovannini, G., \& Feretti, L. 2004, J. Korean Astron. Soc., 37, 323

Govoni, F., Enßlin, T. A., Feretti, L., \& Giovannini, G. 2001a, A\&A, 369,441

Govoni, F., Feretti, L., Giovannini, G., et al. 2001b, A\&A, 376, 803
Govoni, F., Markevitch, M., Vikhlinin, A., et al. 2004, ApJ, 605, 695 Kassim, N. E., Clarke, T. E., Enßlin, T. A., Cohen, A. S., \& Neumann, D. M. 2001, ApJ, 559, 785

Kempner, J. C., \& David, L. P. 2004, MNRAS, 349, 385

Liang, H., Hunstead, R. W., Birkinshaw, M., \& Andreani, P. 2000, ApJ, 544, 686

Röttgering, H. J. A., Wieringa, M. H., Hunstead, R. W., \& Ekers, R. D. 1997, MNRAS, 290, 577

Schuecker, P., \& Böhringer, H. 1999, in Proceedings of the Ringberg workshop on Diffuse Thermal and Relativistic Plasma in Galaxy Clusters, ed. H. Böhringer, L. Feretti, \& P. Schuecker, MPE Report, 271, 43

Schuecker, P., Böhringer, H., Reiprich, T. H., \& Feretti, L. 2001, A\&A, 378,408

Valtchanov, I., Murphy, T., Pierre, M., Hunstead, R., \& Lémonon, L. 2002, A\&A, 392, 795

Zhang, Y.-Y., Finoguenov, A., Böhringer, H., et al. 2004, A\&A, 413, 49 